\title{
Numerical Modeling of Silicon Photodiodes for High-Accuracy Applications Part II. Interpreting Oxide-Bias Experiments
}

\section{Jon Geist}

National Institute of Standards and Technology, Gaithersburg, MD 20899

Rainer Köhler and Roland Goebel BIPM, F-92310 Sèvres, France and

A. M. Robinson and C. R. James University of Alberta, Alberta, Canada T6G 2 G7
The semiconductor device modeling program PC-1D and the programs that support its use in high-accuracy modeling of photodiodes, all of which were described in Part I of this series of papers, are used to simulate oxide-bias self-calibration experiments on three different types of silicon photodiodes. It is shown that these simulations can be used to determine photodiode characteristics, including the internal quantum efficiency for the different types of photodiodes. In the latter case, the simulations provide more accurate values than can be determined by using the conventional data reduction procedure, and an uncertainty estimate can be derived. Finally, it is shown that $0.9997 \pm 0.0003$ is a nominal value for the internal quantum efficiency of one type of photodiode over the 440 to $460 \mathrm{~nm}$ spectral region.

Key words: induced junction photodiode; inversion layer photodiode; numerical modeling; numerical simulation; oxide-bias experiment; quantum efficiency.

Accepted: May 29, 1991

\section{Introduction}

Part II of this series of papers presents the results of high-accuracy simulations of various oxide-bias experiments performed on silicon photodiodes. The simulations were carried out by using the semiconductor device modeling program PC-1D ${ }^{1}$ and a set of three programs designed to support the use of PC-1D in this specific application. Both PC-1D and the support programs are described in Part $I$ of this series of papers.

The oxide-bias experiment [1-3] consists of recording the ratio of the photocurrent as a function of oxide-bias voltage to the zero-bias

\footnotetext{
${ }^{1}$ Certain commercial equipment, instruments, or materials are identified in this paper to specify adequately the experimental procedurc. Such identification does not imply recommendation or endorsement by the National Institute of Standard and Technology, nor does it imply that the materials or equipment identificd arc necessarily the best available for the purpose.
}

photocurrent during irradiation of the photodiode with a stable photon flux. The oxide bias is usually applied with a transparent water-drop electrode. A review is given in reference [3].

Part II is organized as follows: Section 2 describes the simulation of an oxide-bias, self-calibration experiment on an EG\&G UV444B type photodiode. The purpose of this simulation is to demonstrate the derivation of an accurate value for the zero-bias quantum efficiency including an uncertainty estimate. Section 3 describes the simulation of an oxide-bias experiment on a Hamamatsu 1337 type photodiode. The purpose of this simulation is to determine nominal values for the charge trapped in the oxide covering the front surface of the photodiode, and for the surface recombination velocity at the oxide-silicon interface. Section 4 describes the simulation of an oxide-bias experiment on a 
UDT UV100 type photodiode. The purpose of this simulation is to determine a nominal high-accuracy value for the internal quantum efficiency of this type of photodiode and the nominal wavelength region where this value is valid. Section 5 gives the conclusions of Part II.

\section{EG\&G UV444B Photodiode}

The EG\&G UV444B silicon photodiode was the first of its type to be self-calibrated by using the oxide-bias experiment [2]. This type of photodiode consists of a $300 \mu \mathrm{m}$ thick, $300 \Omega \mathrm{cm}$, phosphorusdoped, $n$-type silicon substrate with an $n^{+}$-type phosphorus diffusion in the rear surface, a $p^{+}$-type boron diffusion in the front surface, and a nominally $120 \mathrm{~nm}$ thick, thermally grown oxide-passivation layer over the front surface. Figure 1 shows the front-region doping profile that was obtained from spreading resistance measurements on a representative UV444B photodiode. Figure 1 also shows the equilibrium hole concentration calculated for this doping distribution by using PC-1D.

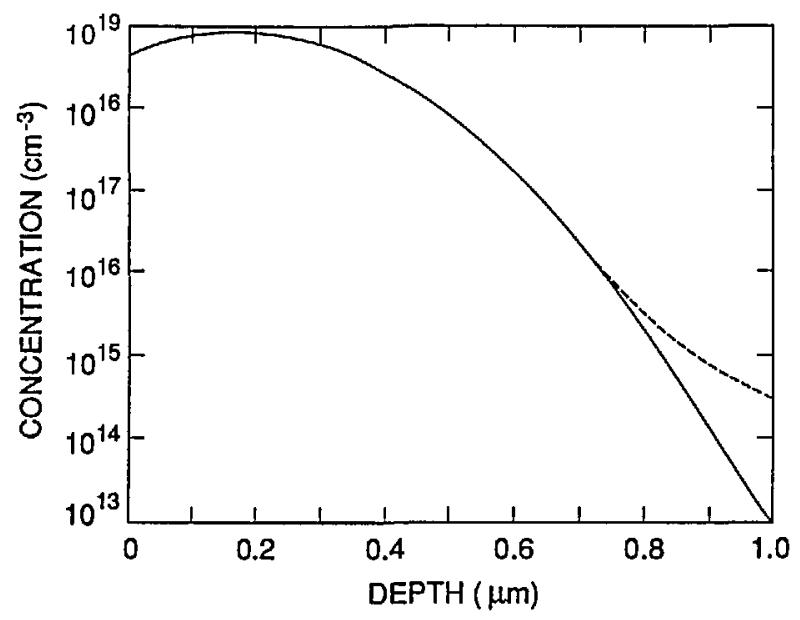

Figure 1. The front-region boron concentration (continuous linc) and the equilibrium hole concentration (dashed line) calculated by PC-1D for a typical EG\&G UV444B type photodiode.

The rear-region, $n^{+}$-type diffusion in the UV444B was not modeled; instead it was assumed that the recombination velocity at the rear of the device was zero. This assumption is not physically realizable, but it results in a mirror for minority carriers at the rear of the device, just as does the $n^{+}$-type diffusion. This assumption is useful because it requires fewer finite elements, thereby leaving more of them available for modeling the front region and depletion region.
Figure 2 compares simulated and experimental results of an oxide-bias experiment on a UV444B photodiode for irradiation by a $3 \mathrm{~mm}$ diameter spot of $476.2 \mathrm{~nm}$ laser radiation through a water-drop electrode. Reference [4] describes the apparatus used to obtain the experimental data. The absorption-coefficient data in the file SIL_WEAK.ABS used for this simulation were calculated from eq [5] that was fit to the data measured by Weakliem and Redfield [6]. These data are described in Part I. Both the surface recombination velocity $S$ and the number density $N_{\mathrm{ss}}$ of the oxide fixed charge were adjusted in the simulation to obtain a good fit to the experimental data in figure 2 .

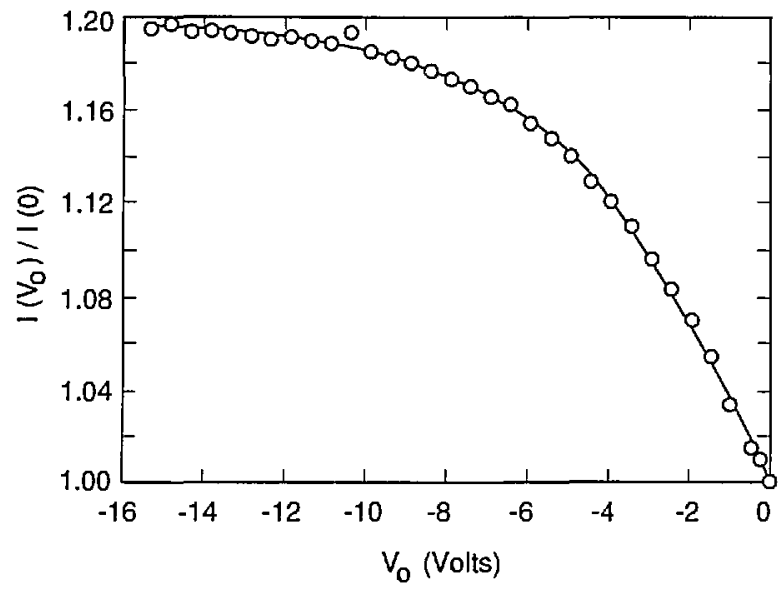

Figure 2. Ratio of the photocurrent $I\left(V_{0}\right)$ as a function of oxide-bias voltage $V_{0}$ to the photocurrent $I(0)$ at zero-bias voltage as measured on an EG\&G UV444B silicon photodiode and as calculated with PC-1D with a front-surface recombination velocity $S=6000 \mathrm{~cm} / \mathrm{s}$ and an oxide trapped chargc number density $N_{\mathrm{ss}}=1.942 \times 10^{12} \mathrm{~cm}^{-2}$.

The procedure used to fit the simulated data to the experimental data shown in figure 2 was as follows: first, a value of $S$ was chosen, then the value of $N_{\text {ss }}$ was adjusted until the average residual of the fit to the photocurrent ratio was zero for the last two data points in the figure. This gave a value of $N_{\text {ss }}$ for each value of $S$ that was chosen. The best combination of $S$ and $N_{\mathrm{ss}}$ was then obtained by minimizing the residual standard deviation over all of the data shown in figure 2.

Figures 3, 4, and 5 plot the residuals (differences) between the results of the fits and the experimental results, and table 1 lists the residual standard deviation for three different choices of $S$ and $N_{\mathrm{ss}}$. It is clear by inspection of the figures that 
the combination of $S$ and $N_{\mathrm{ss}}$ used for figure 3 gives a better overall fit than either of the other choices, even though the residual standard deviation for figure 3 is not very much lower than that for figure 4 or figure 5 . One reason for the relatively small decrease in residual standard deviation with the best fit is the effect of the apparent outlier points near -1 and $-10.5 \mathrm{~V}$ oxide bias.

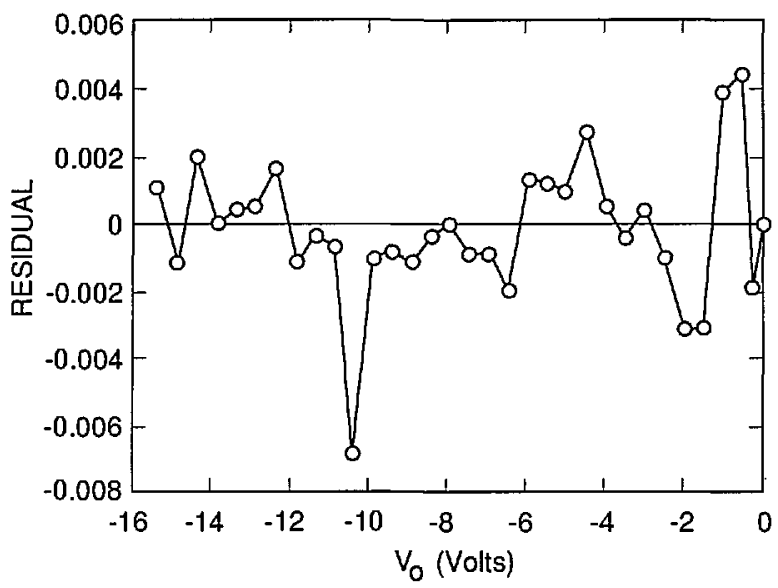

Figure 3. The residuals between the experimental data of figure 2 and the simulated data calculated by PC-1D when $S=6000 \mathrm{~cm} / \mathrm{s}$ and $N_{\mathrm{ss}}=1.942 \times 10^{12} \mathrm{~cm}^{-2}$.

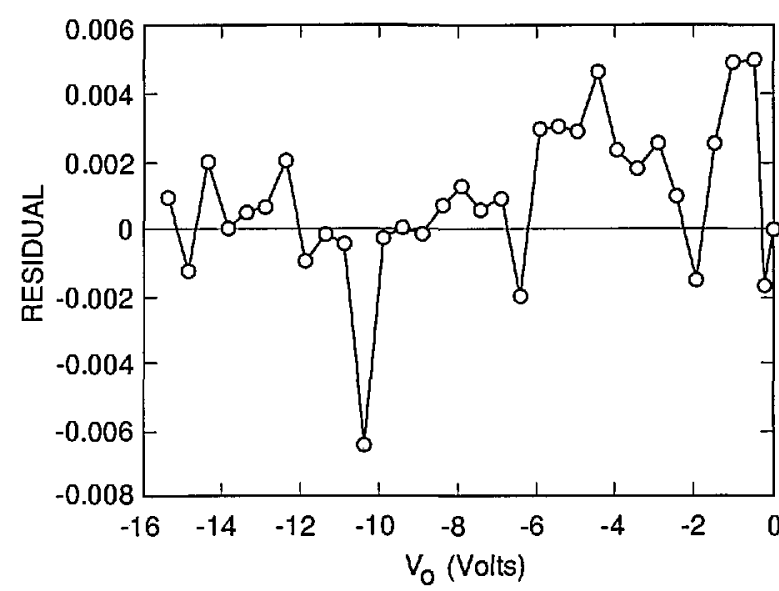

Figure 4. The residuals between the experimental data of figure 2 and the simulated data calculated by PC-1D when $S=5000 \mathrm{~cm} / \mathrm{s}$ and $N_{\mathrm{ss}}=2.011 \times 10^{12} \mathrm{~cm}^{-2}$.

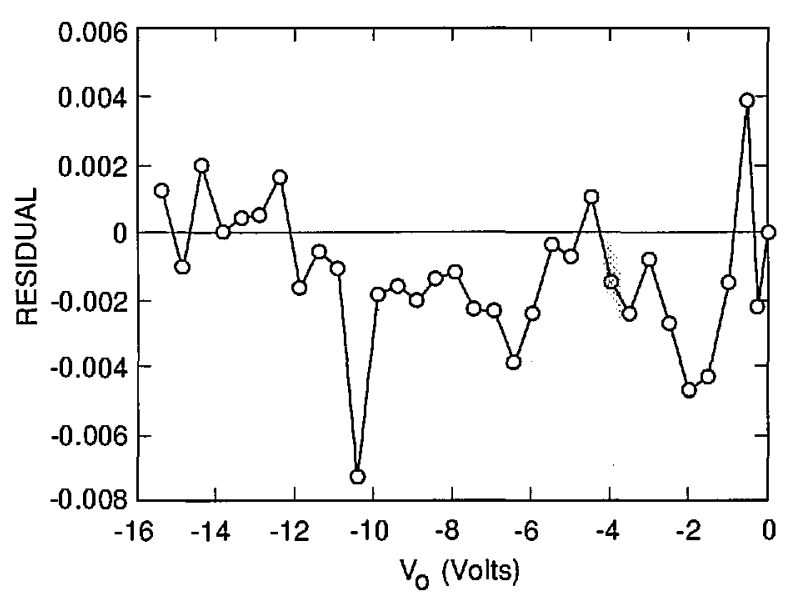

Figure 5. The rcsiduals between the experimental data of figure 2 and the simulated data calculated by PC-1D when $S=7000 \mathrm{~cm} / \mathrm{s}$ and $N_{\mathrm{ss}}=1.883 \times 10^{12} \mathrm{~cm}^{-2}$.

Table 1, Comparison of simulated oxide bias results with expcrimental results for a UV444B type photodiode where Res. std. dev. is the standard deviation of the difference between simulated and experimental results

\begin{tabular}{ccc}
\hline$S(\mathrm{~cm} / \mathrm{s})$ & $N_{\mathrm{ss}}\left(10^{12} \mathrm{~cm}^{-3}\right)$ & Res. std. dev. \\
\hline 5000 & 2.011 & 0.002233 \\
6000 & 1.942 & 0.002076 \\
7000 & 1.883 & 0.002093 \\
\hline
\end{tabular}

The assignment of $S=6000 \mathrm{~cm} / \mathrm{s}$ and $N_{\mathrm{ss}}=$ $1.942 \times 10^{12} \mathrm{~cm}^{-2}$ with estimated uncertainties of $\pm 1000 \mathrm{~cm} / \mathrm{s}$ and $\pm 0.070 \times 10^{12} \mathrm{~cm}^{-2}$, respectively, is justified by figures 3,4 , and 5 . This assignment corresponds to an internal quantum deficiency (one minus the internal quantum efficiency) at zero oxide bias of $0.1690 \pm 0.0021$. The stated uncertainty is the maximum difference between the internal quantum deficiency calculated for the combination of $S$ and $N_{\mathrm{ss}}$ used in figure 3 , and those calculated for the combinations of $S$ and $N_{\mathrm{ss}}$ used for figures 4 and 5 .

The assumption that the average of the last two data points in figure 3 corresponds to $100 \%$ internal quantum efficiency gives a zero-bias internal quantum deficiency of 0.1634 with no reliable way to estimate the uncertainty associated with that value. This assumption, which is usually used with the oxide-bias, self-calibration experiment, produces a result that differs from the more accurate value determined from the fit by 0.0056 . This difference is about $3.3 \%$ of the effect being calculated, and more than 2.5 times the uncertainty associated with the more accurate value. 
It must be emphasized that the results reported here are illustrative rather than definitive since the doping distribution data in figure 1 were obtained from one photodiode, and the oxide-bias data in figure 2 were obtained from a different photodiode. An additional uncertainty associated with the effect of variations in doping distribution would be needed, or the doping distribution must be measured on the actual photodiode used in the selfcalibration.

Because oxide-bias voltages large enough to nearly saturate the photodiode response are also large enough to change $S$ or $N_{\mathrm{ss}}$, the oxide-bias measurement is already destructive to a certain extent, and it must be carried out as described in reference [7] in order to yield the highest accuracy of which it is capable. Therefore, the sacrifice of the photodiode for a spreading resistance measurement is not as impractical as it might at first seem. Nevertheless, it is inconvenient, and more convenient approaches are being developed [3].

The results reported here might be considered illustrative for another reason. The fitting and uncertainty analysis were carried out by hand in a somewhat subjective manner. Ideally, the fitting and uncertainty analysis would be carried out automatically by a fitting routine incorporated into the set of programs used to run PC-1D for photodiode modeling. However, this task might not be as straightforward as it first would seem because the fit should be constrained to pass through the mean of the last few data points at the highest oxide-bias voltage, as it was in the hand fit reported here. Without this constraint, the minimum residual standard deviation might be obtained for a choice of parameters that clearly does not fit the data well at the highest oxide-bias voltages. It is clear that the data at the highest oxide-bias voltages should be weighted more heavily in the fit, but it is not clear how much more heavily. Therefore, an automated program does not eliminate the subjectivity, but only transfers it to the weighting of the data.

\section{Hamamatsu 1337 Photodiode}

Like the EG\&G UV444B photodiode, the Hamamatsu 1337 is a $p^{+} n n^{+}$photodiode, but its front-region doping profile is shallower, its background $n$-type dopant concentration is $5 \times 10^{12} \mathrm{~cm}^{-3}$, and its oxide-passivation layer has a nominal thickness of $25 \mathrm{~nm}$. Figure 6 shows relative front-region doping profiles for six different Hamamatsu 1337 photodiodes taken from four different batches. The curves in figure 6 were normalized to unity at the oxide-silicon interface because it is the shape of the doping profile rather than the magnitude that has the major effect on the internal quantum efficiency [8]. The measured dopant concentrations at the oxide-silicon interface varied between $2 \times 10^{18}$ and $1 \times 10^{19} \mathrm{~cm}^{-3}$ for the six photodiodes of figure 6 .

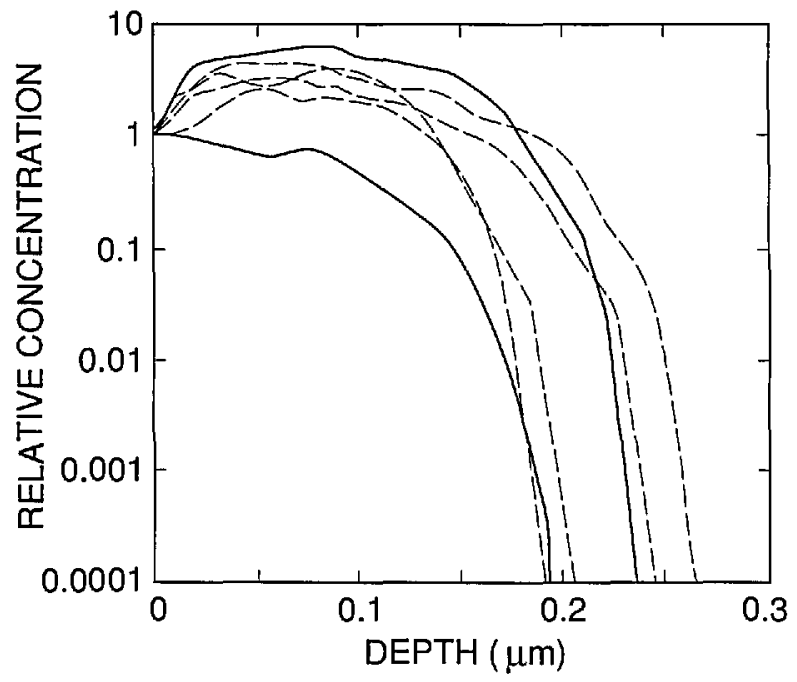

Figure 6. The relative front-region boron concentration for six typical Hamamatsu 1337 type photodiodes. The two profiles shown with solid lines bracket the other four in their effeet on the shape of the internal quantum-deficiency speetrum.

Figure 7 shows the results of modeling an oxidebias experiment on a 1337 type photodiode at $476.2 \mathrm{~nm}$ by using the doping profile that corresponds to the lower solid curve in figure 6 . The fit shown in figure 7 was obtained with $N_{\mathrm{ss}}=-3.0 \times 10^{12} \mathrm{~cm}^{-2}$ and $S=1.835 \times 10^{5} \mathrm{~cm} / \mathrm{s}$, and gave a zero-bias internal quantum deficiency of 0.003199 . A fit that looked virtually identical to that shown in figure 7 was obtained for the doping profile corresponding to the upper solid curve in figure 6 by using $N_{\mathrm{ss}}=-4 \times 10^{12} \mathrm{~cm}^{-2}$ and $S=1.56 \times 10^{5} \mathrm{~cm} / \mathrm{s}$. This fit gave a zero-bias internal quantum efficiency of 0.003280 . As in the case of the UV444B type photodiode, the SIL WEAK.ABS absorption-coefficient data were used, and the rear of the photodiode was modeled as uniformly doped with zero recombination velocity.

Figure 8 compares the internal quantum deficiencies calculated from the doping profile corresponding to the solid curves in figure 6 by using the values of $N_{\mathrm{ss}}$ and $S$ that were obtained from the 


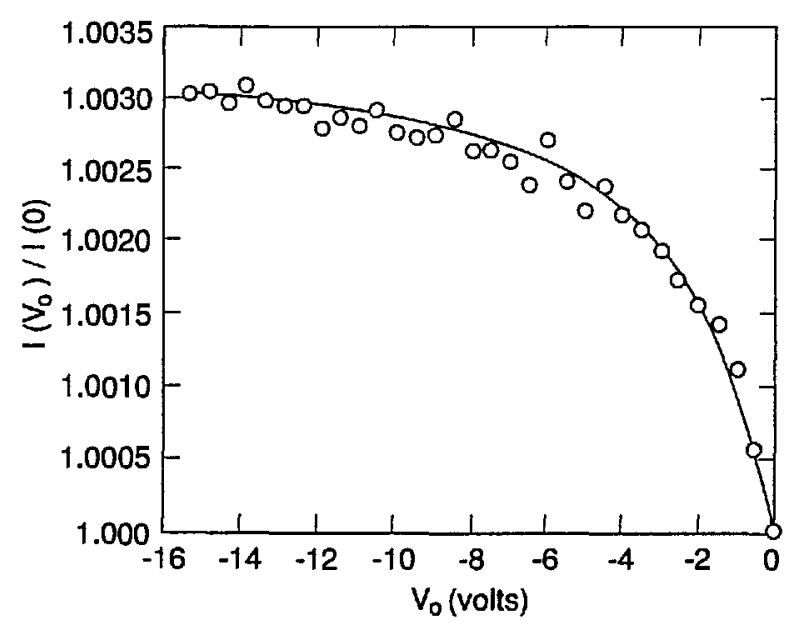

Figure 7. Ratio of the photocurrent $I\left(V_{0}\right)$ as a function of oxide-bias voltage $V_{0}$ to the photocurrent $I(0)$ at zero-bias voltage as measured on a Hamamatsu 1337 silicon photodiode and as calculated with PC-1D for the doping distribution corresponding to the upper solid curve in figure 6 with $S=1.835 \times 10^{5} \mathrm{~cm} / \mathrm{s}$ and $N_{\mathrm{ss}}=-3.0 \times 10^{12} \mathrm{~cm}^{-2}$.

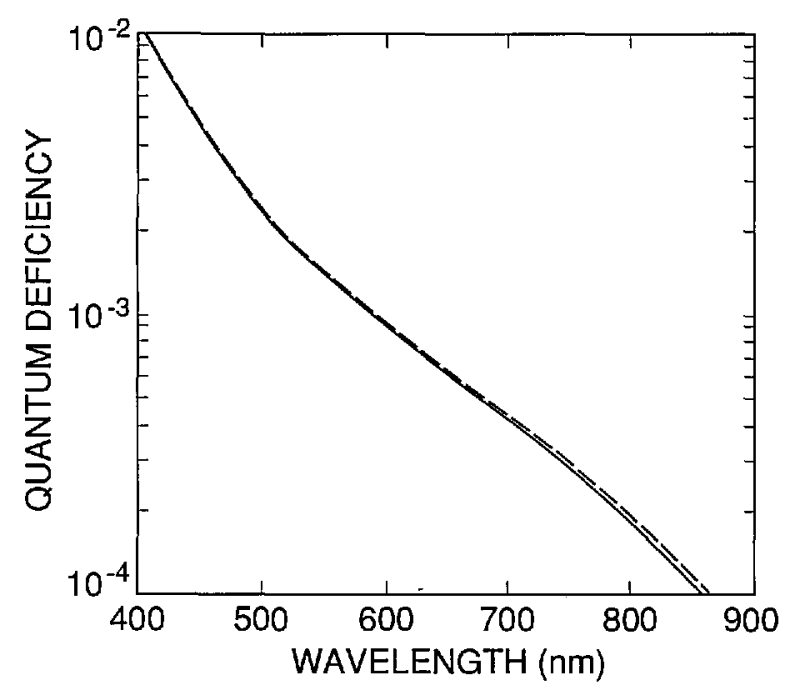

Figure 8. Simulated internal quantum deficiencies for the 1337 type photodiode having the oxide-bias data shown in figure 7 : (solid curve) for the doping profile that corresponds to the lower solid curve in figure 6 with $N_{\mathrm{ss}}=-3.0 \times 10^{12} \mathrm{~cm}^{-2}$ and $S=1.835 \times 10^{5} \mathrm{~cm} / \mathrm{s}$, and (dashed curve) for the doping profile that corresponds to the upper solid curve in figure 6 with $N_{\mathrm{ss}}=-4 \times 10^{12} \mathrm{~cm}^{-2}$ and $S=1.56 \times 10^{5} \mathrm{~cm} / \mathrm{s}$.

simulated oxide-bias experiments based on each of those profiles. The fact that the two curves have very similar shapes is very important. It suggests that a single function of wavelength might be used to extrapolate the internal quantum efficiency of any 1337 type photodiode, rather than a different function for each different photodiode. This possibility is examined in detail in Part III of this series of papers.

Notice that the sign of the trapped charge in the 1337 type photodiode is the opposite of that for the UV444B photodiode, and that the interface recombination velocity is much larger. Both the sign of the trapped charge and the large value of the recombination velocity are consistent with an oxide prepared by chemical vapor deposition rather than by thermal oxidation, because the former is known to produce trapped positive charge and a low surface recombination velocity. The modeling carried out here shows that a large negative charge in the oxide more than makes up for the greater surface recombination velocity by repelling minority carriers from the surface.

The fact that the 1337 type photodiode has negative rather than positive charge trapped in its oxide is important for its possible use in quantum-efficiency interpolation and extrapolation. If the oxide contained a significant amount of positive charge, as assumed in reference [9], the oxide-silicon interface would be depleted of holes, leading to a very large decrease in the energy needed to impactionize a valence band electron. However, since the oxide contains negative charge, the interface is accumulated. In this case, even though the charge density is quite large, there is little or no decrease in the energy needed to impact-ionize a valence band electron [9]. This is important because a large decrease in the energy required to impact-ionize a valence band electron might cause the quantum yield [10] for electron-hole pair production of the silicon near the front surface to exceed unity at wavelengths as long as $450 \mathrm{~nm}$. This would limit the usefulness of this type of photodiode for extrapolating and interpolating quantum-efficiency calibrations. If this limitation were encountered, it could only be overcome with a very accurate model of the quantum yield in the presence of surface fields. Such a model appears well beyond the current state of the art [9].

Even though the uncertainties in the internal quantum deficiency associated with the diodeto-diode variations in doping profile are quite small for the 1337 type photodiode, the oxide-bias experiment is still less than ideal for self-calibration [3]. However, since the 1337 is so well suited for interpolating and extrapolating internal quantumdeficiency data, as shown in Part III, all that is needed are ways to calibrate the photodiode that work over limited spectral regions near $450 \mathrm{~nm}$ and near $900 \mathrm{~nm}$. The next section illustrates how 
simulation can be used to predict the internal quantum efficiency of a different type of photodiode with an uncertainty of about 0.0003 at $450 \mathrm{~nm}$.

\section{UDT UV100 Photodiode}

The UDT UV100 type photodiode is usually assumed to have an internal quantum efficiency of unity in the short wavelength portion of the visible [11]. In fact, this assumption is the basis for the use of the QED 100 and QED 200 multiple reflection radiometers [12], which contain modified UV100 photodiodes, as high-accuracy absolute radiometric standards.

As the state of the art in absolute radiometry improves with time, the assumption of unity quantum efficiency for the UV100 type photodiode becomes more questionable. What is needed is a nominal internal quantum-deficiency spectrum accompanied by a reliable uncertainty spectrum. Since the 1337 type photodiode can be used to interpolate or extrapolate internal quantumefficiency calibrations, as shown in Part III, it is only necessary to have these spectra over a limited spectral region above $400 \mathrm{~nm}$. (Below $400 \mathrm{~nm}$, the nonunity quantum yield would be a problem.) One way to obtain these spectra is to calculate them from nominal characteristics of the UV100 type of photodiode. The pertinent characteristics can be obtained by fitting numerical simulations of oxidebias measurements to experimental results as demonstrated in the preceding sections.

The UV100 type of photodiode is quite different from the UV444B and 1337 type photodiodes. Its $n^{+} p$ junction is not formed by introducing a frontregion dopant, but is induced in a $100 \Omega \mathrm{cm} p$-type (approximately $1.3 \times 10^{14} \mathrm{~cm}^{-3}$, boron-doped) silicon substrate by the growth of an oxide passivation layer having a large concentration of trapped positive charge [13].

Figure 9 compares the results of simulated and experimental oxide-bias experiments on a UV100 type photodiode. Here again, the absorptioncoefficient data in SIL WEAK.ABS were used, and the rear of the photodiode was modeled as uniformly doped with a surface recombination velocity of zero. Since the UV100 type photodiode is an $n^{+} p$ type rather than a $p^{+} n$ type, the effect of increasing negative bias is to decrease rather than increase the photocurrent. However, because the zero-bias quantum efficiency is so close to unity, this procedure samples the portion of the curve with the most information about the values of $N_{\mathrm{ss}}$ and $S$, as can be seen in the figure.

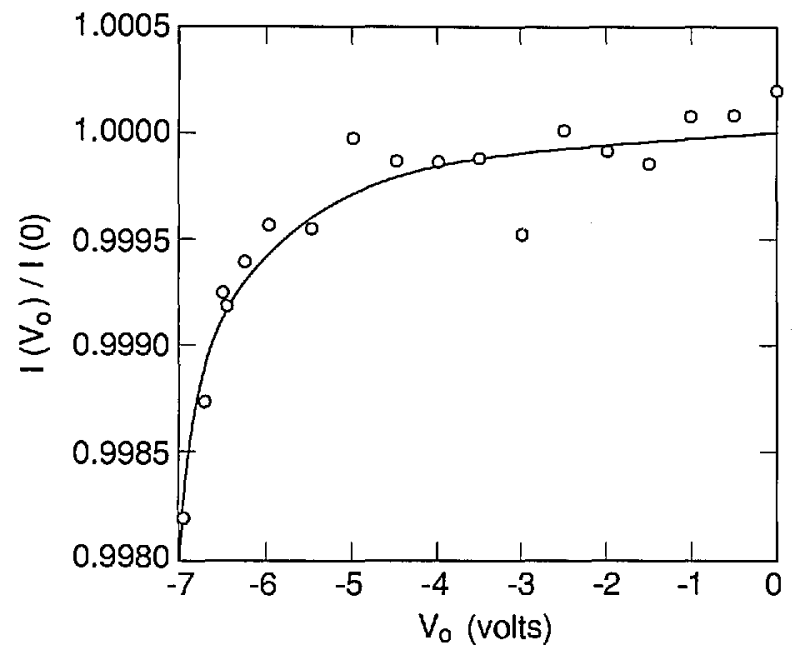

Figure 9. Ratio of the photocurrent $I\left(V_{0}\right)$ as a function of oxide-bias voltage $V_{0}$ to the photocurrent $I(0)$ at zero-bias voltage as mcasured on a typical UDT UV100 silicon photodiode and as calculated with PC-1D for $S=3.0 \times 10^{5} \mathrm{~cm} / \mathrm{s}$ and $N_{\mathrm{ss}}=1.38 \times 10^{12} \mathrm{~cm}^{-2}$.

These values of $N_{\mathrm{ss}}$ and $S$ are used to simulate the internal quantum-deficiency spectra for a nominal UV100 photodiode in figure 10 . The three different curves shown in figure 10 correspond to minority carrier lifetimes $\tau$ of $1 \mu \mathrm{s}, 10 \mu \mathrm{s}$, and $81 \mathrm{~ms}$, respectively, in the rear region of the photodiode. The lifetimes of 1 and $10 \mu s$ give internal quantum deficiencies at $700 \mathrm{~nm}$ that bracket the range measured for six typical UV100 type photodiodes [14], and the $81 \mathrm{~ms}$ lifetime is the longest lifetime that the default silicon material model of PC-1D will allow for $100 \Omega \mathrm{cm}, p$-type silicon with a recombination trap at mid-gap.

The region between the curves corresponding to $\tau=1$ and $\tau=10 \mu \mathrm{S}$ in figure 10 is a nominal internal quantum-deficiency spectrum that applies to UV100 type photodiodes and to QED 100 and QED 200 radiometers at zero reverse bias $[3,12]$. Figure 11 compares the spectrum for $\tau=1 \mu \mathrm{s}$ around its minimum in the 400 to $500 \mathrm{~nm}$ spectral region with spectra obtained for the cases where either the front-region surface recombination velocity is doubled, or the positive charge trapped in the oxide is halved. These are conservative limits to associate with the mass production of photodiodes in a controlled planar-silicon fabrication process. These should also be conservative limits for the effects of environmental stress, such as humidity, temperature, and irradiation with ultraviolet radiation, for UV100 photodiodes [15-16] made after 1986. 


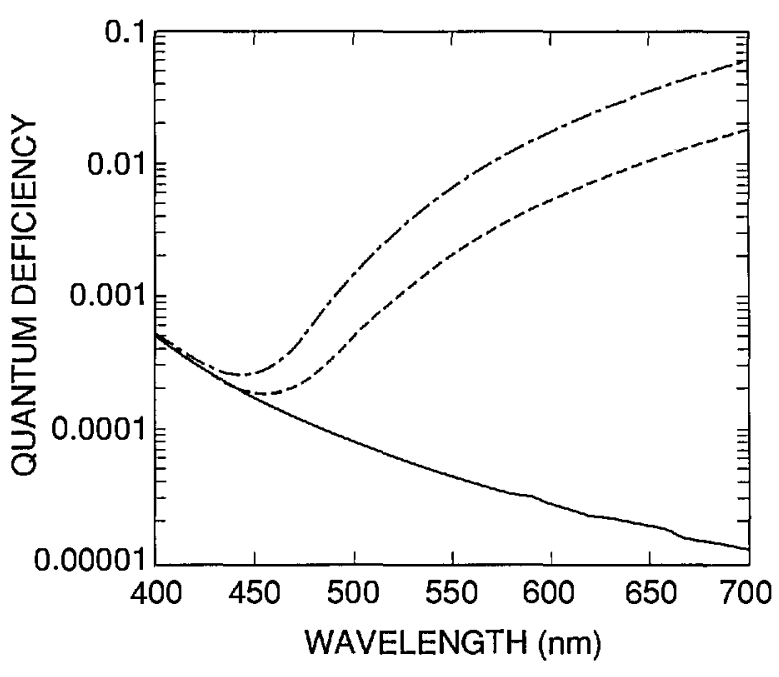

Figure 10. Internal quantum-deficiency spectra calculated by PC-1D for $S=3.0 \times 10^{5} \mathrm{~cm} / \mathrm{s}, N_{\mathrm{ss}}=1.38 \times 10^{12} \mathrm{~cm}^{-2}$, and rearregion lifetimes $\tau$ of $81 \mathrm{~ms}$ (solid line), $10 \mu \mathrm{s}$ (dashed line), and $1 \mu \mathrm{s}$ (dot-dashed line).

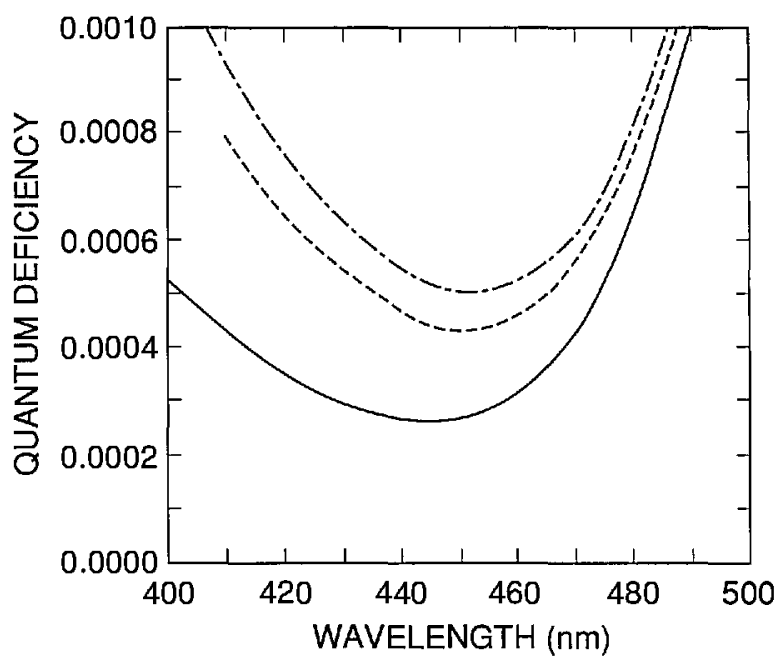

Figure 11. Internal quantum-deficiency spectra calculated by PC-1D for a rear-region lifetime $\tau=1 \mu$ sith $S=3.0 \times 10^{5} \mathrm{~cm} / \mathrm{s}$ and $N_{\mathrm{ss}}=1.38 \times 10^{12} \mathrm{~cm}^{-2}$ (solid line), with $\tau=1 \mu \mathrm{s}$ with $S=6.0 \times 10^{5} \mathrm{~cm} / \mathrm{s}$ and $N_{\mathrm{ss}}=1.38 \times 10^{12} \mathrm{~cm}^{-2}$ (dashed line), and with $\tau=1 \mu \mathrm{s}$ with $S=3.0 \times 10^{5} \mathrm{~cm} / \mathrm{s}$ and $N_{\mathrm{ss}}=0.69 \times 10^{12} \mathrm{~cm}^{-2}$ (dot-dashed line).

The curves in figure 11 can be used to estimate uncertainties to be associated with the nominal values of the internal quantum efficiency of the UV100 type photodiode. Summing in quadrature the differences between the nominal spectrum and the other two spectra in figure 11 produces a somewhat conservative estimate since increases in surface recombination velocity tend to be correlated with increases in trapped charge. In the region from 440 to $460 \mathrm{~nm}$, a nominal value of
$0.0003 \pm 0.0003$ is a practical summary of the results of such an analysis. This result should be considered a one-standard-deviation equivalent, rather than a limit of error, since the whole analysis is based on the results obtained for a single device. Outside the 440 to $460 \mathrm{~nm}$ spectral region, the uncertainties grow so rapidly that they are not really practical for high-accuracy applications.

There are a number of sources of error not yet considered, but since their effect scales with the magnitude of the internal quantum deficiency, they are all negligible with respect to \pm 0.0003 . This is illustrated in figure 12 for the uncertainty associated with the absorption coefficient of silicon in the 440 to $460 \mathrm{~nm}$ spectral region, which is the largest uncertainty not considered so far.

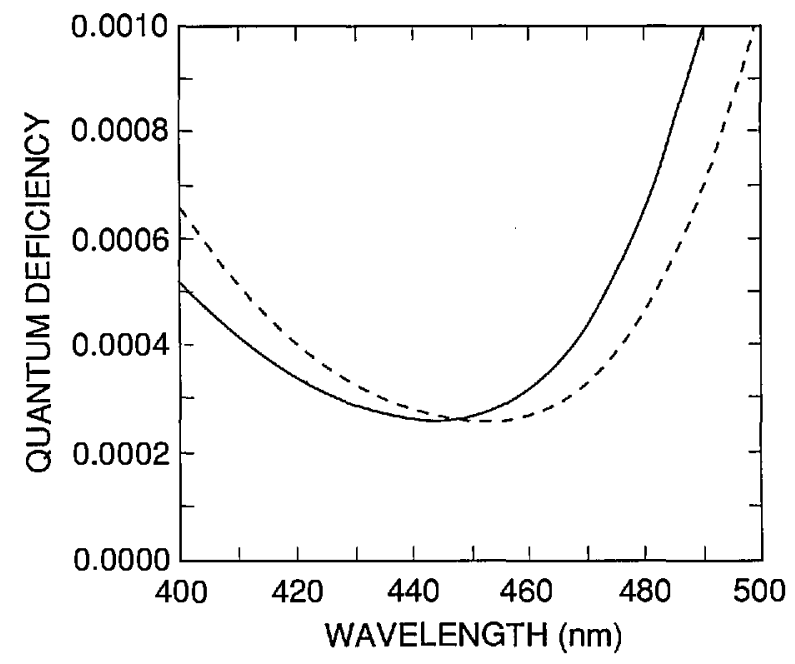

Figure 12. Comparison of the internal quantum-deficiency spectra calculatcd for a UV100 type photodiode for a rearregion lifetime $\tau=1 \mu \mathrm{s}$ with $S=3.0 \times 10^{5} \mathrm{~cm} / \mathrm{s}$ and $N_{\mathrm{ss}}=1.38 \times 10^{12} \mathrm{~cm}^{-2}$ with the absorption-coefficient data in the SIL WEAK.ABS file (solid line), and with the absorptioncoefficient data in the SIL PHIL.ABS filc (dashed line). The data in these files arc described in more detail in Part I of this series of papers.

Figure 12 compares the spectrum of figure 11 for $\tau=1 \mu \mathrm{s}$, which was based on the absorptioncoefficient data in SIL_WEAK.ABS, with the same spectra calculated for identical parameters, but based on absorption-coefficient data (measured by Philipp [17], and described in Part I) that is stored in SIL_PHIL.ABS. When added in quadrature to the \pm 0.0003 assigned to the variations in $N_{\mathrm{ss}}$ and $S$ from diode to diode, the effect of the uncertainty in the absorption coefficient is negligible. This might not remain true if a much larger wavelength interval were considered. 
Because of the large long-wavelength quantum deficiency shown in figure 10 , reverse bias is often applied to UV100 photodiodes and QED 100 and 200 radiometers when they are used at the longer wavelengths. By moving the depletion region toward the rear of the photodiode, the reverse bias forces the internal quantum deficiency to approach the lower quantum deficiency limit given by the 81 $\mathrm{ms}$ curve in figure $10[12,18-19]$. Reverse bias is also applied to eliminate the saturation type nonlinearity that is sometimes observed with photocurrents above a few microamperes. However, recent results [4] suggest that moderate bias levels can cause gain, resulting in an internal quantum efficiency in excess of unity. This effectively precludes the application of moderate reverse bias in highaccuracy applications.

Figure 13 plots a simulation of the decrease in the internal quantum deficiency as a function of reverse bias for the UV100 type photodiode at the 442, 476, and $514 \mathrm{~nm}$ laser lines. The feature in the curves for reverse-bias voltages less (more negative) than $-3.5 \mathrm{~V}$ is a symptom of a problem with PC-1D. It may mean that more than 150 finite elements are needed to maintain the level of accuracy achieved as the reverse bias is increased, but this is not certain. In any case, it not only invalidates the data for reverse-bias voltages more negative than $-3.5 \mathrm{~V}$, but it also casts some doubt on any conclusions drawn about the data at the less negative bias voltages as well. At best, figure 13 suggests that $480 \mathrm{~nm}$ is a practical upper limit to the wavelength at which reverse bias can be used to eliminate the effect of recombination in the rear region of the photodiode without risking gain. However, the best advice would be never to use a UV100 photodiode or QED radiometer with a bias voltage without first assuring experimentally that gain is not a problem.

\section{Conclusion}

Part II of this series of papers has demonstrated the use of Version 2 of PC-1D.EXE, RUN_PC1D.BAT, MAKE_PRM.EXE, and READ_PDF.EXE to simulate various oxide-bias experiments of interest to high-accuracy applications of silicon photodiodes. In each case, the simulation was used to interpret an oxide-bias experiment, with different sorts of information being sought from the different experiments.

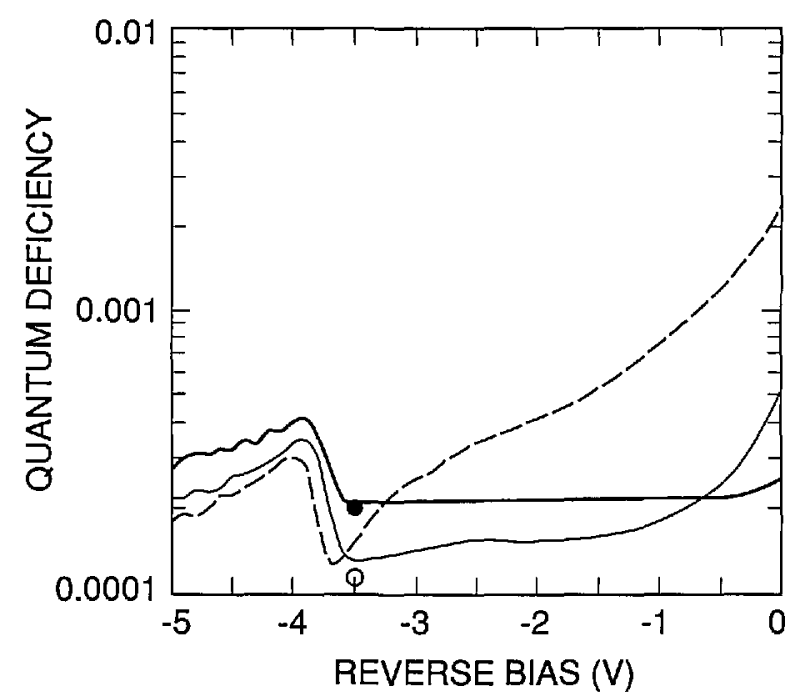

Figure 13. Simulation of the decrease in internal quantumdeficiency of a UV100 type photodiode with reverse bias at $442 \mathrm{~nm}$ (heavy solid line), $476 \mathrm{~nm}$ (light solid line), and $514 \mathrm{~nm}$ (dashed line). The values of $N_{\mathrm{ss}}, S$, and $\tau$ are as in figure 12. The feature near $-3.5 \mathrm{~V}$ is an artifact of the simulation. The open circle and closed circle are the internal quantum deficiencies at $442 \mathrm{~nm}$ and $476 \mathrm{~nm}$, respectively, for $\tau=81 \mathrm{~ms}$. The internal quantum deficiency at $514 \mathrm{~nm}$ for the same condition is too small to appear on the graph.

It was shown that the simulations can be used to derive a more accurate internal quantumdeficiency value from an oxide-bias experiment than that available from the conventional data reduction, and that an uncertainty can be associated with the value derived from the simulation based on how well the simulated oxide-bias data can be fitted to the experimental data. It was also shown that simulations can be used to determine nominal values for the front-surface recombination velocity and for the charge trapped in the frontsurface oxide, and that values for these parameters can be used to determine nominal internal quantum-deficiency spectra for various types of silicon photodiodes.

As well as illustrating these general ideas, some more specific results were also obtained. First, it was shown that a nominal internal quantum deficiency of $0.0003 \pm 0.0003$ is appropriate for the unbiased UDT UV100 type photodiode in the 440 to $460 \mathrm{~nm}$ spectral region. Second, it was shown that the shape of the internal quantum-deficiency spectrum of the 1337 type photodiode is quite independent of typical variations in doping profile. This result suggests that the 1337 type photodiode might serve to extrapolate high-accuracy calibrations 
obtained from cryogenic absolute radiometers or from QED type radiometers in the 440 to $460 \mathrm{~nm}$ spectral region to longer wavelengths with little or no loss of accuracy. Part III of this series of papers shows that this is the case. Finally, it was shown that reverse-bias simulations based on the programs described in Part I of this series of papers do not work well enough with inversion layer (induced junction) photodiodes to be useful for highaccuracy applications.

About the authors: Jon Geist is a physicist in the NIST Semiconductor Electronics Division, Rainer Köhler and Roland Goeble are physicists at the Bureau International des Poids et Mesures in Sèvres, France, while A. M. (Sandy) Robinson and C. R. (Bob) James are Professors of Electrical Engineering at the University of Alberta in Edmonton, Alberta, Canada. Prof. James is presently Vice-President (Research) at the University.

\section{References}

[1] Geist, J., J. Appl. Phys. 51, 3993 (1980).

[2] Zalewski, E. F., and Geist, J., Appl. Opt. 19, 1214 (1980).

[3] Geist, J., SPIE 1109, 246 (1989), provides a review of the oxide-bias, self-calibration technique.

[4] Köhler, R., Pello, R., and Bonhoure, J., Appl. Opt. 29, 4212 (1990).

[5] Geist, J., Migdall, A., and Baltes, H. P., Appl. Opt. 27, 3777 (1988).

[6] Weakliem, H. A., and Redfield, D., J. Appl. Phys. 50, 1491 (1979), and personal communication.

[7] Kcy, P. J., Fox, N. P., and Rastello, M. L., Metrolgia 21, 81 (1985).

[8] Geist, J., and Baltes, H. P., Appl. Opt. 28, 3929 (1989).

[9] Geist, J., Gardner, J. L., and Wilkinson, F. J., Phys. Rev. 42B, 1262 (1990).

[10] Geist, J., and Wang, C. S., Phys. Rev. 27B, 4841 (1983).

[11] Gcist, J., Liang, E., and Schaefer, A. R., J. Appl. Phys. 52, 4879 (1981).

[12] Zalewski, E. F., and Duda, C. R., Appl. Opt. 22, 2867 (1983).

[13] Hansen, T., Phys. Scr. 18, 471 (1978).

[14] Houston, J. M., and Saunders, R. B., personal communication of data.

[15] Korde, R., and Geist, J., Solid-State Electronics 30, 89 (1987).

[16] Korde, R., and Geist, J., Appl. Opt. 26, 5284 (1987).

[17] Philipp, H. R., J. Appl. Phys. 43, 2835 (1972), and personal communication.

[18] Geist, J., Zalewski, E. F., and Schaefer, A. R., Appl. Opt. 19, 3795 (1980).

[19] Schaefer, A. R., Zalewski, E. F., and Geist, J., Appl. Opt. 22, 1232 (1983). 